\title{
A descriptive research audit of the long term developmental outcomes of 30 infants with Hypoxic Ischemic Encephalopathy (HIE) who received hypothermia at a secondary Hospital in South Africa.
}

\author{
*Suzanne Stark, B.OT (US), MSc. OT (UCT). http://orcid.org/0000-0003-2 I l 6-9887 \\ Occupational Therapist, Carpe Diem School, George, South Africa \\ Desiree Van der Vyver, B.OT (US). http://orcid.org/0000-0002-4532-2279 \\ Chief Occupational Therapist, Department of Health, George Provincial Hospital, Western Cape. \\ Pamela Gretschel, B.OT (US), M ECI (UP), PHD OT (UCT). https://orcid.org/0000-0002-7890-3635 \\ Senior Lecturer, Department of Health and Rehabilitation Sciences, Faculty of Health Sciences University of Cape Town.
}

Introduction: Hypothermia is a neuroprotective strategy proven to reduce death and disability in children presenting with Hypoxic Ischemic Encephalopathy (HIE). Little evidence exists regarding the long term developmental outcomes of infants who received hypothermia within a secondary hospital in South Africa.

Method: Drawing on a descriptive research audit this paper describes the development (up to five years) of 30 infants with HIE who received hypothermia at the George hospital in South Africa. Developmental outcomes were measured at 3, 9, 12, 24 and 36 months using the Strive Towards Achieving Results Together (START) and at 48 and 60 months using the Early Childhood Developmental Criteria (ECDC). Summary data (variables considered risk factors) were collected retrospectively. The associations between this data and developmental outcomes are described.

Results: Twenty children presented with typical development, eight presented with cerebral palsy (CP) and two were lost to follow up. A significant association between a severe HIE score and CP was found $(p=0.0005)$. The children performed equally in fine motor, gross motor and cognitive abilities at four years with poorer outcomes in cognitive and fine motor abilities recorded at five years. The perceptual activity both age groups struggled with the most was directionality.

Conclusion: The results of the research audit support the need for long-term occupational therapy follow-up of children with HIE to monitor their development and provide specific interventions to promote their development and prevent barriers to learning. This paper also advocates the need for occupational therapists working in the public health sector to have access to standardised assessment tools which allow them to closely monitor the development of infants who present with HIE at birth.

Key words: Hypoxic Ischemic Encephalopathy, hypothermia, developmental outcomes, environment

\section{INTRODUCTION}

George hospital, a secondary hospital in the Western Cape Province, collaborates with Groote Schuur Hospital, a tertiary institution, to provide input and guidance in the use of hypothermia for newborn infants presenting with Hypoxic Ischemic Encephalopathy (HIE). In November 2009 hypothermia was introduced at the George Hospital and the two occupational therapists working at the hospital were requested to assist with evaluating the developmental outcomes of these infants. To date, research evidence has firmly established that hypothermia is effective in the reduction of death and disability amongst infants with $\mathrm{HIE}^{1,2,3}$ but the long-term developmental outcomes of infants who received hypothermia are still uncertain. This study, which commenced in January 2010 , describes the long-term (up to five years of age) developmental outcomes of 30 infants with HIE who received hypothermia at the George Hospital. Data collection was completed in March 2019.

This study was in many ways unique in its approach. The study tracked the development of children who received hypothermia at a secondary hospital in a low-resource public health setting. All participants were classified as falling in the low socio-economic bracket, receiving an annual income of less than R40 000 and R50 000 for single and family incomes respectively ${ }^{4}$. This is in contrast to the majority of studies undertaken in tertiary hospital settings ${ }^{5}$. This study is also one of a few South African studies tracking the developmental outcomes of children beyond two years of age. While occupational therapists in South Africa have been involved in monitoring the developmental outcomes of infants who received hypothermia, these studies have focused on monitoring isolated aspects of development (e.g. gross motor development) ${ }^{6}$. The current study adopted a holistic approach to monitoring development in the domains of gross motor, fine motor, speech and language as well as cognition.

This research audit aimed to answer the research question: What are the long term developmental outcomes of infants with HIE who received hypothermia at birth? The specific objectives of this study were: 
To measure and describe the developmental outcomes of infants with HIE who received hypothermia at the George Hospital, at the ages of 3, 9, 12, 24, 36, 48 and 60 months.

To document summary data (variables considered risk factors for $\mathrm{HIE})$ and describe their association with developmental outcomes.

\section{LITERATURE REVIEW}

\section{Hypoxic ischemic encephalopathy (HIE)}

Perinatal asphyxia takes place when the new born is delayed in establishing spontaneous respiration following delivery ${ }^{7}$. HIE is the term used to describe the neurological syndromes which can occur following perinatal asphyxia" ${ }^{7}$. Perinatal asphyxia is a common cause of death in children in South Africa ${ }^{8}$, Signs of asphyxia at birth contribute to approximately $23 \%$ of the 4 million neonatal deaths and $8 \%$ of all deaths in children under five years of age throughout the world ${ }^{7}$. In developed countries, death or disability has been recorded in $53 \%$ to $61 \%$ of infants diagnosed as presenting with moderate to severe $\mathrm{HIE}^{9,10}$. Various studies have found that $\mathrm{HIE}$ following birth asphyxia contributes greatly to neonatal mortality and morbidity including long-term neurodevelopmental disability in up to $25 \%$ to $60 \%$ of survivors ${ }^{1,10,11}$.

\section{The HIE Score}

The HIE score, also known as the Thompson score, is a clinical assessment tool consisting of clinical signs that are closely associated with dysfunction of the Central Nervous System (CNS) ${ }^{12}$. It is used to assess the status of the infant following birth asphyxia ${ }^{13}$. A score between I and I0 indicates mild HIE, a score between II and I5 is interpreted as moderate HIE and a score between 15 and 22 is considered as severe $\mathrm{HIE}^{12,13}$. Various studies have shown that the Thompson score can be used as a prognostic tool to predict the long-term neurodevelopmental and morbidity outcome in asphyxiated term newborns undergoing controlled hypothermia, with a higher score significantly associated with an adverse outcome ${ }^{14}$.

\section{Hypothermia}

Hypothermia is a neuroprotective strategy where the core body temperature of a full term infant is reduced by 3 to 4 degrees within 6 hours of birth. The process of hypothermia is sustained for 72 hours before gradual re-warming commences ${ }^{15}$. A Cochrane review published in September 2013 included I I randomised controlled trials comprising of I 505 term and late preterm infants with moderate or severe $\mathrm{HIE}^{16}$. This review showed that the use of hypothermia resulted in a statistically significant and clinically important reduction in the combined outcome of death or serious disability at 18 months of age. A distinction is made between whole body cooling where the rectal temperature is kept at $33-34^{\circ} \mathrm{C}$ and selective head cooling where cooling of only the head (and the brain) is targeted and the rectal temperature is kept $I^{\circ} \mathrm{C}$ higher $\left(34-35^{\circ} \mathrm{C}\right)^{17}$. Based on South African research, hypothermia has been described as a feasible treatment to use in resource-limited settings when done in accordance with a strict protocol ${ }^{18}$. The protocol used by the George Hospital at the time that the study commenced in 2010 , is described in Table I.

\section{Long-term developmental outcomes following HIE and hypothermia}

Various studies have advocated for the long-term follow up of child developmental outcomes to determine the long-term effects of hypothermia ${ }^{1,2,3}$. This is imperative as it is known that subtle
Table I: Summary of criteria for selective lead cooling at George Hospital, South Africa

The diagnosis of Neonatal Encephalopathy is defined by:

- Any clinical signs of encephalopathy and clinical seizures or clinical signs of moderate- severe HIE in 3 or more of the categories on the Thompson Score.

- $\quad$ Suspected intrapartum hypoxia suggested by either base deficit of 16 or more in the first hour of life on cord or infant blood, or an 10 minute Apgar of less than 7, or the infant requiring assisted ventilation at age 10 minutes.

\begin{tabular}{|l|l|}
\hline $\begin{array}{l}\text { Criteria for selective } \\
\text { head cooling }\end{array}$ & $\begin{array}{l}\text { Contra-indications for selective } \\
\text { head cooling }\end{array}$ \\
\hline $\begin{array}{l}\text { The gestational age } \\
\text { of the infant should } \\
\text { be } 36 \text { weeks or more } \\
\text { and the weight of the } \\
\text { infant should be more } \\
\text { than I } 800 \text { grams } \\
\text { Hypothermia of the in- } \\
\text { fant has to be initiated } \\
\text { by } 6 \text { hours of age. }\end{array}$ & $\begin{array}{l}\text { - The infant is unlikely to benefit } \\
\text { outcome. }\end{array}$ \\
\end{tabular}

neuro-developmental disabilities such as perceptual and learning difficulties often only become apparent at school going age ${ }^{19}$. A study by Shankaran, Pappas, McDonald et al. ${ }^{20}$ on the cognitive, attention, executive function and visio-spatial abilities of 122 of the participants aged 6 to 7 years revealed attention-executive dysfunction in $4 \%$ of children receiving hypothermia and $13 \%$ of those receiving usual care. Visio-spatial dysfunction occurred in $4 \%$ and $3 \%$ respectively ${ }^{20}$. Another study reported on the neurological outcomes, cognitive, attention, executive and visio-spatial function as well as physical and psychosocial health of 190 children $^{21}$. The two primary outcomes namely death and an Intelligence Quotient (IQ) $<70$, were noted among $47 \%$ of the hypothermia group and $62 \%$ of the control group. Secondary outcomes included a mortality rate of 28 to $44 \%$, death or CP of 41 to $60 \%$ and death and severe disability of $4 \mathrm{I}$ to $60 \%$ in the hypothermia and control groups respectively ${ }^{21}$. A study performed in Western Australia reported on the outcomes of 65 infants who received hypothermia. Thirteen had mild, 35 had moderate and 17 had severe HIE. Nine of the 13 infants with mild HIE were followed up and had no disability. Among the 52 infants with moderate to severe HIE, there were nine deaths. Data on the developmental outcomes were available for 39 of the infants who survived. The incidence of severe disability for this group was $23 \%^{22}$.

\section{METHODS}

\section{Details of the audit}

All term infants born at George Hospital from January 2010 to March 2014 presenting with HIE who received hypothermia were included in the research audit. In view of time, staff and cost constraints the study was able to accommodate 30 participants who would be able to attend follow up appointments at George Hospital.

\section{Measuring instruments}

Based on the literature reviewed, the researchers identified developmental assessments frequently used to determine developmental 
outcomes of infants presenting with HIE following birth. These include the Bayley Scales, Vineland Adaptive Behaviour Scale, Griffiths and Peabody Motor scales'. Working in a context with restricted access to resources, these developmental assessments were however not available to the researchers. The researchers therefore used tools available to them in the occupational therapy department at George Hospital. The Strive Towards Achieving Results Together (START) was used to assess developmental outcomes from birth to three years and the Early Childhood Developmental Criteria (ECDC) was used to assess cognitive, fine motor and gross motor developmental outcomes at 4 and 5 years of age. Children with $C P$ were assessed by means of the Gross Motor Function Classification System- Expanded and Revised (GMFCS-E\&R). The researchers followed the definition for cerebral palsy, as described by Rosenbaum, Paneth and Levinton et al., as "a group of permanent disorders of the development of movement and posture, causing activity limitation, that are attributed to non-progressive disturbances that occurred in the developing foetal or infant brain"23:9.

The START is a developmental assessment and intervention programme developed by the Sunshine Foundation in Johannesburg, South Africa by Solarsh, Katz, Goodman ${ }^{24}$. Its main purpose is to assist caregivers in the identification of areas of development that are potentially problematic. Four developmental areas are assessed, namely gross motor development, fine motor development, communication and activities of daily living (ADL). Since the tool is not designed as a measuring instrument, there is no published data available on the validity and reliability of the START. As no numerical values are attached to the various developmental areas assessed, the researchers adapted the START by assigning values. If a child is able to, for example, lift his/her head while in prone at the age of 3 months the child was assigned a score of I. Should the child not be able to perform this task, the child was assigned a score of 0 . In this this study, the START was used to assess the developmental outcomes of children from 0 months to 3 years at the following ages: 3, 9, 12, 18, 24 and 36 months.

The GMFCS - E\&R is based on self-initiated movement, with emphasis on sitting, transfers, and mobility ${ }^{25}$. The aim of the GMFCS $-E \& R$ is to assess a child or young person's abilities and limitations relating to gross motor function in everyday life ${ }^{25}$. It consists of a five-level classification system which indicates the person's ability to mobilise. Level I indicates good mobility whereas Level $V$ indicates severely impaired mobility.

The ECDC is a set of developmental scales developed by a South African occupational therapist, Ingrid Herbst ${ }^{26}$, with the specific purpose of evaluating the extent to which children living in environmentally disadvantaged contexts have accomplished cognitive and motor developmental tasks underlying school readiness. The test gives special attention to relevance and aptness according to culture and was developed due to scarcity of standardised tests appropriate to the South African context ${ }^{26}$. The ECDC test comprises three subdivisions: cognitive aspects, fine motor co-ordination and gross motor skills ${ }^{26}$. With coefficients of $r=0,85$ and $r=0,93$ for the cognitive and motor subsections respectively, the test showed high test-retest reliability. The inter-rater reliability of the test was investigated and correlations of 0,99 were obtained for both the cognitive and combined motor scores of the scales. Component and factor analyses were conducted to establish the validity of the scales. The results supported the construct validity of the scales. The norm group is based upon the data of more than 900 children receiving and not receiving educational stimulation ${ }^{26}$.

Summary data (gender of participants, Apgar scores, HIE scores, possible reasons for HIE, HIV exposure as well as the age of the mother at the time of the infant's birth) was collated from the medical folders retrospectively. These variables were included following a review of the medical literature on HIE and hypothermia describing the absence or presence of these variables as risk factors for developmental delay.

\section{Analysis}

The outcomes from the summary data sheet yielded nominal variables. Developmental outcomes as well as certain outcomes from the summary data sheet are expressed as discrete variables. The three authors carefully checked the data collected to identify errors. By examining the graphic display of data, the researchers decided which measures to use to summarise and describe the categorical and numerical variables ${ }^{27}$. The researchers analysed the data relating to outcomes as measured with the START by drawing stem-and-leaf plots of every area of development evaluated at every age. Based on the stem-and-leaf plots, it was evident that, in all areas of development across all age groups, the data were skewed towards high scores. The authors therefore decided to use the median rather than the mean to express the data. By implication the children were able to perform the developmental tasks expected of them. The total scores of each area of development differed from each other and between the various age groups. The researchers therefore expressed the median as a percentage to allow for comparisons between the different areas of development in the various age groups. Statistical analysis was performed with the Fischer's Exact Test by grouping data in 2 by 2 tables. A p-value of less than $p<0.05$ was regarded as statistically significant.

\section{Ethics}

Ethical clearance was granted by the Human Research Ethics Committee from the Faculty of Health Sciences at the University of Cape Town (HREC REF number is $26 \mathrm{I} / 20$ I2). Institutional permission was granted by the Medical Manager of the George Hospital in March 2012 and the Health Research component from the Western Cape Department of Health granted the study a reference number (RP99) in 2012.

\section{RESULTS}

\section{Summary Data}

Associations between summary data variables and developmental outcomes are presented in Table II (on page 38). A severe HIE score indicated a significant likelihood of CP, with a p-value of 0.0005 .

The possible reasons for HIE as recorded in the medical folders are listed in Table III (on page 38). Prolonged stage 2 labour accounted for II out of 30 of the possible reasons for HIE.

The ages of the mothers at the time of the babies' birth is depicted in Figure I (on page 38). Seven mothers were younger than 20 years and two thirds of the mothers were between 21 and 25 years of age. The average birth weight of the babies was 3317 grams with the lowest weight at 2320 grams and the highest weight at 4360 grams. The median weight was 3280 grams. Only full term babies received hypothermia.

\section{Developmental data}

Two of the 30 children were permanently lost to follow up, one passed away on day six following birth and one child was lost to follow-up after birth. When measured with the START, the overall developmental data of 28 children indicated that eight presented 
Table II: Associations between summary data and developmental outcomes using the Fischer's Exact Test

\begin{tabular}{|c|c|c|c|}
\hline \multirow{2}{*}{$\begin{array}{l}\text { Summary data variable } \\
\text { Gender }(n=28)\end{array}$} & \multicolumn{2}{|c|}{ Developmental outcome } & \multirow{2}{*}{$\frac{\text { Level of significance }}{\text { p-value }}$} \\
\hline & Typical Development & Cerebral Palsy & \\
\hline Male $(n=14)$ & 9 & 5 & $p=0.68$ \\
\hline Female $(n=14)$ & II & 3 & \\
\hline \multicolumn{4}{|l|}{ HIE score $(n=28)$} \\
\hline Mild HIE and Moderate HIE & 19 & 2 & \multirow[t]{2}{*}{${ }^{*} \mathrm{p}=0.0005$} \\
\hline Severe HIE & 1 & 6 & \\
\hline \multicolumn{4}{|l|}{$\begin{array}{l}\text { Apgar at } 5 \text { minutes } \\
(n=28)\end{array}$} \\
\hline Score I-5 & 15 & 5 & $p=0.65$ \\
\hline Score 6-10 & 5 & 3 & \\
\hline \multicolumn{4}{|l|}{ Apgar at 10 minutes $(n=27)$} \\
\hline Score I-5 & 6 & 4 & $p=0.4 I$ \\
\hline Score 6-10 & 13 & 4 & \\
\hline RVD exposed $(n=4)$ & 4 & & \\
\hline
\end{tabular}

Table III: Possible obstetric reason for HIE as recorded in the medical folder

\begin{tabular}{|l|l|}
\hline \multicolumn{2}{|l|}{ Possible reason recorded for HIE in medical folder $(\mathbf{n}=\mathbf{3 0})$} \\
\hline & $\mathbf{n}(\%)$ \\
\hline Prolonged stage 2 labour & $\mathrm{I}(36.7 \%)$ \\
\hline Meconium stained liquor & $5(16.7 \%)$ \\
\hline Abruptio placenta & $4(13.3 \%)$ \\
\hline Foetal distress & $2(6.7 \%)$ \\
\hline Variable decelerations & $\mathrm{I}(3.3 \%)$ \\
\hline Poor respiratory effort & $\mathrm{I}(3.3 \%)$ \\
\hline Breach delivery & $\mathrm{I}(3.3 \%)$ \\
\hline Placenta previa & $\mathrm{I}(3.3 \%)$ \\
\hline Late decelerations & $\mathrm{I}(3.3 \%)$ \\
\hline Meconium aspiration & $\mathrm{I}(3.3 \%)$ \\
\hline No reason recorded & $\mathrm{I}(3.3 \%)$ \\
\hline Cord around neck & $\mathrm{I}(3.3 \%)$ \\
\hline
\end{tabular}

Age of the mother $n=30$

12

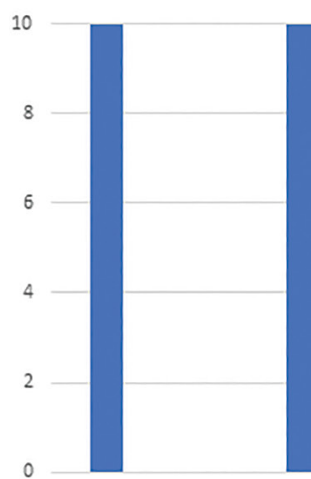

$15 \cdot 20$

$21 \cdot 25$

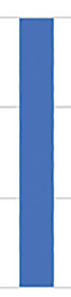

$26 \cdot 30$

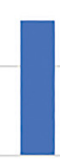

$31-35$

with $\mathrm{CP}$ and 20 children presented with typical development. Of the 28 children who were followed up, five children from the group who demonstrated typical developmental did not attend all of their follow-up appointments. Two were followed up at 12 months, one followed up at 6 months, another followed up until 36 months and the fifth child followed up until 9 months. During these evaluations the children presented with typical development when evaluated with the START. The researchers went on to assume that these five children were likely to continue following a pattern of typical development for the development assessed by the START. The assessment results of the 20 children who presented with typical development (as evaluated with the START) are illustrated in Figure 2 (on page 39).

The median of each developmental area at each age bracket is expressed as a percentage to compare the various age brackets in Figure 2 (page 39). Gross motor development remained consistently high across all the age brackets with a slight decrease at 36 months. This could be attributed to two of the gross motor tasks namely 'pedalling a tricycle' and 'starts to use a bat and ball'. Both of these activities are dependent on play equipment, which many of the children do not have access to due to socio-economic constraints. There was a decrease in fine motor development at 9 months with a return to age expected norms at 12 months and then a gradual decline from 12 months to 36 months. The most common fine motor tasks that children found difficult at 9 months were the ability to pick up and hold 2 objects in one hand and the ability to hold one object in each hand. At 24 months, placing a square and triangle into a form board was singled out as the most common fine motor task that this age group struggled with. This task is dependent on access to a specific educational toy to which many children might not have access. At 36 months, fine motor tasks included more complicated tasks such as snipping with a pair of scissors as well as aspects closely related to cognitive development such as matching colours and pointing to body parts.

Figure I: The age of the mothers at the time of the babies' birth 


\section{START}

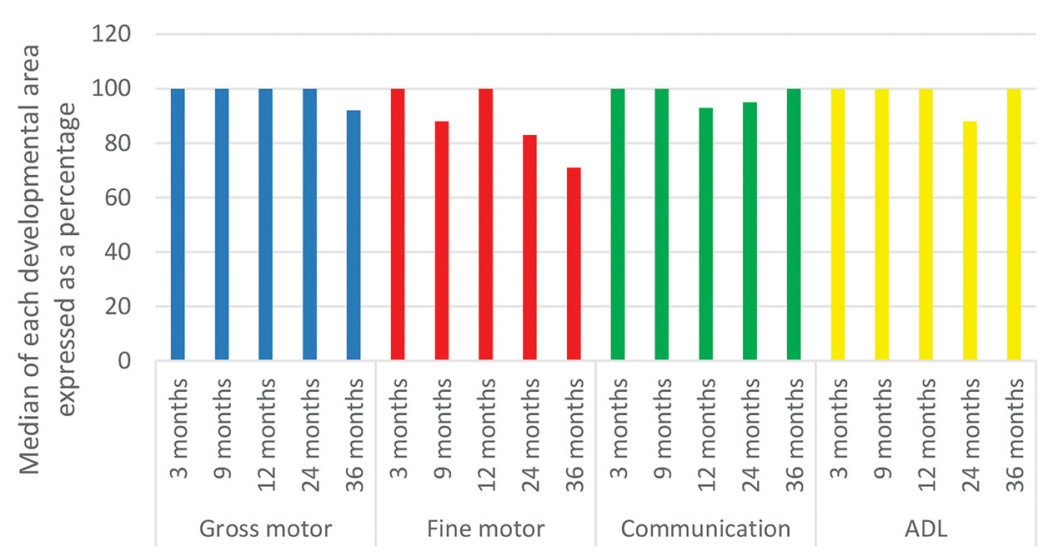

Figure 2: Developmental outcomes of typically developing children when measured with the START

Table IV: The results of the ECDC evaluation at 4 and 5 years

\begin{tabular}{|l|l|l|l|}
\hline Index & Level of performance & 4 years $(n=13)$ & 5 years $(n=15)$ \\
\hline \multirow{3}{*}{ Cognitive } & Below average & $6(46.2 \%)$ & $9(60 \%)$ \\
\cline { 2 - 4 } & Average and above average & $7(53.8 \%)$ & $6(40 \%)$ \\
\hline \multirow{3}{*}{ Fine motor } & Below average & $6(46.2 \%)$ & $9(60 \%)$ \\
\cline { 2 - 4 } & Average and above average & $7(53.8 \%)$ & $6(40 \%)$ \\
\hline \multicolumn{3}{|l}{} \\
\hline \multirow{2}{*}{ Gross motor } & Below average & $6(46.2 \%)$ & $5(33.3 \%)$ \\
\cline { 2 - 4 } & Average and above average & $7(53.8 \%)$ & $10(66.7 \%)$ \\
\hline
\end{tabular}

Table V: A comparative of the ECDC cognitive index components in relation to the number of children who obtained average to above average scores at 4 and 5 years respectively

\begin{tabular}{|l|c|l|c|}
\hline $\begin{array}{c}\text { ECDC Cognitive } \\
\text { Index Components }\end{array}$ & $\begin{array}{c}\text { Number of learner(s) } \\
\text { who obtained an } \\
\text { average or above } \\
\text { average ECDC score } \\
\text { at 4 years (n= 13) }\end{array}$ & $\begin{array}{c}\text { ECDC Cognitive } \\
\text { Index Components }\end{array}$ & $\begin{array}{c}\text { Number of } \\
\text { learner(s) who } \\
\text { obtained an av- } \\
\text { erage or above } \\
\text { average ECDC } \\
\text { score at 5 years } \\
\text { (n= 15) }\end{array}$ \\
\hline Colour concepts & 10 & Colour concept & 12 \\
\hline Form concept & 8 & $\begin{array}{l}\text { Visio- motor } \\
\text { integration }\end{array}$ & 1 I \\
\hline $\begin{array}{l}\text { Picture } \\
\text { interpretation }\end{array}$ & 8 & Picture interpretation & 9 \\
\hline Number concept & 8 & Stick building & 9 \\
\hline Incomplete Man & 7 & Incomplete man & 8 \\
\hline Puzzle & 6 & Block building & 8 \\
\hline $\begin{array}{l}\text { Visio-motor integra- } \\
\text { tion }\end{array}$ & 6 & Puzzle & 7 \\
\hline Stick building & 5 & Form concept & 7 \\
\hline Block Building & 1 & Number concept & 6 \\
\hline Directionality & & Directionality & 5 \\
\hline
\end{tabular}

Varied access to high quality educational settings could have had an impact on this result. The development of communication skills also remained consistently high except for a slight decline at 12 and 24 months. It is likely that the requirements in terms of communication in these two age brackets are high, but that children are able to master communication tasks over a period of time as indicated by the median of $100 \%$ at 36 months.

Children's abilities to perform ADL were consistently high except at 24 months where one of the tasks requires children to be dry during the day. Most children were not yet ready for potty training or had not as yet mastered this task.

At four and five years, the children's development was evaluated using the ECDC. These evaluations were only performed with children who presented with typical development as measured on the START.

The results of the ECDC evaluated at 4 and 5 years are reflected in Table IV (on this page). The results depict the outcome of the ECDC index for cognitive, fine motor and gross motor domains of development. The ECDC does not differentiate between average and above average performance.

At 4 years seven children scored average and above average on all three domains of development. It is interesting to note that the children performed equally on all three domains at 4 years. This changed at 5 years with poorer outcomes on the ECDC Index and fine motor development and a better score on gross motor outcomes. More children also scored below average on fine motor outcomes and the ECDC Index at 5 years $(n=9)$ than at 4 years $(n=6)$.

The ECDC cognitive index consists of ten subdivisions. When looking at the number of children who obtained an average and above average score on the ECDC Index at 4 and 5 years, it is evident that they struggled more with particular activities. The activities listed in Table $\mathrm{V}$ (on this page) are arranged in order of those which most children mastered to those with which most children struggled with at 4 and 5 years.

Colour concept was the activity both age groups performed the best in, while directionality emerged as the activity in which both groups performed the poorest.

Visual perceptual tasks such as visio-motor integration, stick and block building and picture interpretation improved between 4 to 5 years. Children struggled more with number and form concepts at 5 years when compared to their performance at 4 years.

The GMFCS is used to describe the 
Table VI: GMFCS level and HIE Score

\begin{tabular}{|l|l|}
\hline $\begin{array}{l}\text { GMFCS level of CP children and } \\
\text { description }(\mathbf{n}=\mathbf{8})\end{array}$ & HIE score of child \\
\hline Level I $(n=2)$ & Severe HIE Score $(n=I)$ \\
\cline { 2 - 2 } $\begin{array}{l}\text { Least impairment in terms of mobil- } \\
\text { ity }\end{array}$ & Moderate HIE Score $(n=I)$ \\
\hline $\begin{array}{l}\text { Level } 5(n=6) \\
\text { Most severe impairment in terms of } \\
\text { mobility }\end{array}$ & Mild HIE Score $(n=I)$ \\
\cline { 2 - 2 } & \\
\hline
\end{tabular}

development of the children with cerebral palsy. The GFMCS level gives an indication of the degree of functional mobility impairment. Six of the eight children presenting with CP were classified as Level 5 (most severe impairment in terms of mobility) on the GMFCS. Of the eight children who presented with CP, two children (both on level $V$ of the GMFCS) passed away at the ages of 3 years 6 months and 5 years 3 months respectively. The families of both children informed the researchers that their children passed away, but the causes of death were not shared and were not recorded in the medical files. The GMFCS levels are described in Table VI (above) and highlight the association between a severe HIE score and a high GMFCS level.

\section{DISCUSSION AND IMPLICATIONS}

\section{Summary data}

An analysis of the demographic and summary data showed that neither gender nor Apgar score (a tool to evaluate a new-born's health) showed a statistically significant association with developmental outcomes. The current research concluded that severe HIE scores associated significantly with $C P(p=0.0005)$. This aligns with the results of a South African study by Kali, Martinez-Biarge, van Zyl et al. ${ }^{18}$ which similarly showed that severe HIE and an abnormal MRI were associated with death and severe impairment.

In this study prolonged stage 2 labour accounted for most instances ( $36 \%$ ) as possible reason for HIE compared to the South African study by Horn ${ }^{28}$ where the meconium stained liquor was recorded in most cases (39\%) followed by abnormal foetal heart rate in $36 \%$ of cases and prolonged stage 2 labour in $20 \%$ of infants with HIE. It appears that the peri-partum complications differ from study to study. It is dependent on the doctor's objective description at the time of labour ${ }^{28}$.

The current research further supports the results of previous studies, which have shown that infants who present with a mild HIE score tend to demonstrate normal development ${ }^{21}$. The study by $\mathrm{Pin}^{19}$ indicated that a moderate HIE score is indicative of varied developmental outcomes. While this study found that two out of three children with moderate HIE scores presented with normal development, these numbers are too small to draw conclusions.

Of the four children who were RVD exposed, two demonstrated normal development and two were diagnosed with cerebral palsy. Le Doare ${ }^{29}$ found that in early infancy to 2 years, children who were HIV exposed at birth, did not demonstrate any global delay, however during the preschool years these children had subtle cognitive, motor function, expressive and receptive language deficits. By implication, these children would require long-term, individual follow up assessment sessions to monitor their development and provide intervention, focusing on their affected perceptual, cognitive, speech and language, and fine motor abilities. These underlying performance components form the basis of scholastic abilities such as reading, writing and arithmetic.

\section{Developmental outcomes}

Given the South African context where children in low-resource settings are typically exposed to outdoor play for a large part of the day, the authors are not surprised by the sequence of development of skills based on the results of the ECDC. At 5 years, gross motor development seemed to be developed best followed equally by fine motor development and cognitive development. This is in keeping with the South African study of Sukha ${ }^{6}$ who found that infants who presented with moderate HIE at birth had fewer delays in gross motor skills than with fine motor skills when measured at $I$ and 2 years. Another South African study by Munro, Van Niekerk, and Seedat ${ }^{30}$ also commented on betterthan-expected gross motor skills in South African children from disadvantaged contexts. These authors observed that children from disadvantaged backgrounds are more likely to engage in unrestricted play in open spaces such as the streets of their communities. This kind of play, combined with a lack of access to technology such as computer and video games, contribute to improved gross motor skills. The South African results seem to be in contrast to developed countries such as the United Kingdom where fine motor skills seems to be better developed than gross motor skills ${ }^{31}$. The authors suggest that South African occupational therapists should consider tapping into the strength of gross motor outcomes as a foundation to build and expand the development of cognitive, perceptual and fine motor skills. Acknowledging that more research is needed the South African researchers, Sherry and Draper ${ }^{32}$ postulate a promising link between gross motor interventions during preschool years and the subsequent development of cognitive, visual perceptual and fine motor skills.

When interpreting the results of the performance of the children on the subdivisions of the ECDC Index at four years, the mastery of particularly activities requiring spatial perceptual abilities seem to be problematic. Research has shown that spatial perceptual abilities are related to visio-motor integration (VMI) and required for reading, writing, spelling and arithmetic. While the relationship between VMI and academic achievement is not clearly understood ${ }^{31}$ it is significantly correlated with a child's ability to perform academic work at school $^{33}$. At five years, these difficulties are less apparent although number and form concepts seems to be more difficult and may have subsequent negative implications on progress of arithmetic skills. Directionality was most problematic with both age groups.

The poorer results of the five year old children (when compared to the four year old children) on the cognitive index could be indicative of the long-term effect of HIE on cognition. Perez et al. ${ }^{34}$ concluded that children who sustained HIE without major disability had an increased risk of long-term intellectual, verbal and motor deficits. The authors are careful to attribute poor cognitive and perceptual performance solely to the effects of HIE. It stands to reason that the well documented ripple effect of poverty on early childhood development, co-occurring with risk factors (such as HIE) could culminate in a poorer developmental trajectory of young children ${ }^{32}$.

\section{STRENGTHS AND LIMITATIONS}

\section{Assessment Tools}

When comparing the results of the START evaluation with the ECDC it is evident that study participants performed better on the START than on the ECDC. Four possible reasons will be offered and discussed. Firstly, the START was not originally designed to be an assessment tool, but structured as such by the authors 
of this article. In doing so, its properties may have changed. The authors work as clinicians in a secondary hospital where validated and reliable assessment tools used and described in literature are not available due to reasons described previously. Using valid and reliable assessment tools between 0 and 3 years would no doubt have improved the rigor of the results and made comparisons to previous studies more valid. Sukha ${ }^{5}$, an Occupational Therapist who followed up on the motor outcomes of infants with moderate and severe HIE at a tertiary hospital in South Africa, commented that mild developmental delays are sensitive to standardised assessment tools and these subtle delays are often overlooked with non-standardised assessments. Using standardised assessments will assist clinicians to detect the specific nature of developmental delays in infants with HIE. The authors argue that having access to standardised assessment tools for the age band between 0 and 3 years would greatly contribute to enabling active contributions towards evidence-based practice in terms of early childhood development benchmarking and intervention strategies.

A second possible reason for the discrepancy between outcomes of the START and ECDC evaluation is the age group of the population being evaluated. While the ECDC relies on activities presented to the child in a prescribed manner, the START relies to a large degree on a parent's report of the child's abilities which is subjective, possibly even inaccurate and can question the credibility of the results.

Thirdly, there are certain complexities when awarding a point according to a child's ability to perform a certain activity in the absence of a manual guiding the therapist performing the assessment. For example when using the START if a child, for instance, is able to initiate head control but unable to sustain it, considerations had to be made as to whether to award the point. This could definitely be considered as a limitation to the results of the study and strengthens the argument for the necessity to use standardised assessment tools.

Fourthly, the ECDC can be considered as a more complex tool in terms of the activities that children are required to perform. While both the START and the ECDC evaluate fine and gross motor development, the ECDC evaluates cognition in greater depth and detail than some of the activities of the START which requires underlying cognitive skills.

\section{Other Strengths and limitations}

This study represents research at the level of a secondary hospital conducted by clinical occupational therapists as opposed to the majority of studies undertaken by large tertiary institutions. The holistic approach to development incorporating different spheres of development as well as a keen understanding of the children's' medical background combined with the extended period of follow up contributes to the strength and uniqueness of the study. The findings are not only unique in its context but also an example of a research audit which can be undertaken by other occupational therapists working in the field.

An obvious limitation to the research is the small size of the study which limits the generalisability of the results. The lack of exact socio-economic data and educational level of the parents could also have had a bearing on results. Other factors which could have affected results, and should be considered in future research, is the access of the child to quality early childhood education. This is a major area of interest and research in South Africa as it is considered a key intervention in shaping the future of young children ${ }^{32}$.

The summary data were collected retrospectively and as such, there are incidences where attrition affected the data.

A lack of resources and standardised assessment tools is a common threat to the quality and ongoing monitoring of the effectiveness of occupational therapy services within the government sector in South Africa. It is imperative for occupational therapists to not only understand the value of using standardised assessment tools, but also have the skills to lobby for the procurement of these tools. In addition, occupational therapists should continue working towards validating standardised assessments for use in the South African context.

\section{Acknowledgements}

The authors would like to thank all the medical staff in ward B3 at the George Hospital, particularly Dr Els for her belief in the contribution that Occupational Therapy bring to the quality of life of these children. The authors are sincerely grateful to the parents and children who participated in this study.

\section{Funding}

Funding was received from Ruth Watson OTASA research grant.

\section{REFERENCES}

I. Sculzke SM, Rao S, Patole SK. A systematic review of cooling for neuroprotection in neonates with hypoxic ischemic encephalopathy - are we there yet? BMC Paediatrics. 2007; 7(30). https://doi.org/10.1 186/147I-2431-7-30

2. Shah PS, Ohlsson A, Perlman M. Hypothermia to treat neonatal hypoxic ischemic encephalopathy. Arch paediatric adolensc med. 2007; 16(I0): 95I - 958. https://doi.org/10.1001/archpedi.161.10.95I

3. Blackmon LR, Stark AR. Hypothermia: A Neuroprotective therapy for neonatal hypoxic-ischemic encephalopathy. Paediatrics. 2006; I I7(3): 942 - 948. https://doi.org/10.1542/peds.2005-2950

4. Western Cape Health Services Fees Act. The regulations, prescribing the uniform patient fee schedule for health care services rendered by the Department for externally funded and subsidised patients, issued in terms of the Hospital Ordinance, 1946 (Ordinance 18 of 1946). Provincial Gazette Extraordinary, 19 December 2008. http://www.wcpp.gov.za

5. Padayachee N, Ballot DE. Outcomes of neonates with perinatal asphyxia at a tertiary academic hospital in Johannesburg, South Africa. SAJCH. 2013; 7: 3. https://doi.org/I0.7/96/sajch.574

6. Sukha N. The developmental motor outcomes of infants with Hypoxic Ischaemic Encephalopathy II and III between the ages of 12-24 months at Chris Hani Baragwanath Academic Hospital. Unpublished Master's Thesis; 2013. [cited 2017 April]. Available from: http://wiredspace.wits.ac.za/jspui/bitstream/l 0539//4453/l/ The\%20developmental\%20motor\%20outcomes\%20of\%20 infants\%20with\%20Hypoxic\%20lsc.pdf

7. Mwakyusa SD, Manj KP, Massawe AW. The hypoxic ischaemic encephalopathy score in predicting neurodevelopmental outcome among infants with birth asphyxia at the Muhimbili National Hospital, Dar-es-Salaam, Tanzania. Journal of Tropical Pediatrics. 2009; 55(I): 8 - 14. https://doi.org//0.1093/tropej/fmn06I

8. Pattinson RC, ed. Saving Babies 2008-2009: Seventh Report on Perinatal Care in South Africa. http://www.pipp.co.za/downloads [cited 20 I4 April]. https://doi.org/10.1016/s0 140-6736(09)6। I23-5

9. Gluckman PD, Wyatt JS, Azzopardi D, et al. Selective head cooling with mild systemic hypothermia after neonatal encephalopathy: Multicentre randomized trial. Lancet. 2005; 365: 663 - 670. https://doi.org/10.1016/s0140-6736(05)70932-6 
10. Shankaran S, Woldt E, Koepke T, Bedard MP, Nandyal R. Acute neonatal morbidity and long-term central nervous system sequelae of perinatal asphyxia in term infants. Early Human Development. 1991; 25 (2): 135 - |48. https://doi.org/10.1016/03783782(91)90191-5

II. Robertson CM, Finer NN, Grace MG. School performance of survivors of neonatal encephalopathy associated with birth asphyxia at term. Journal of Paediatrics. 1989; II4: 753 - 760. https://doi.org/10.1016/s0022-3476(89)80132-5.

12. Bhagwani DK, Sharma M, Dolker S, Kothapalli S. To study the correlation of Thompson scoring in predicting early neonatal outcome in post asphyxiated term neonates. J Clin Diagn Res. 2016; 10 (I I): 16 - 19. https://doi.org//0.7860/jcdr/20I6/22896.8882

13. Thompson CM, Puterman AS, Linley LL, Hann FM, van der Elst CW, Molteno CD, Malan AF. The value of the hypoxic ischaemic encephalopathy score in predicting neurodevelopmental outcome. Acta Paediatrica. 1997; 86: 757 - 6I. https://doi.org/I0.1 I I I/j.165I-2227.1997.tb0858I.

14. Mendler MR, Mendler I, Hassan MA, Mayer B, Bode H, Hummler $H D$. Predictive value of Thompson-Score for long-term neurological and cognitive outcome in term newborns with perinatal asphyxia and hypoxic-ischemic encephalopathy undergoing controlled hypothermia treatment. Neonatology. 2018; I14: 34I - 347. https://doi.org//0.1159/00049072

15. Azzopardi D, Brocklehurst P, Edwards D, Halliday H, Levene M, Thoresen M, Whitelaw A. The toby study. Whole body hypothermia for the treatment of perinatal asphyxia encephalopathy: A randomized controlled trail. BMC Paediatrics. 2008; 8: 17. https://doi.org/10.1 I86/I47|-2431-8-I7

16. Thoresen M, Tooley J, Liu X, Jary S, Fleming P, Luyt K, Jain A, Cairns P, Harding D, Sabir H. Time is brain: starting therapeutic hypothermia within three hours after birth improves motor outcome in asphyxiated newborns. Neonatology. 2013: 104(3): 228 - 233. https://doi.org/10.1 I59/000353948

17. Atici A, Celik Y, Gulasi S, Turhan AH, Okuyaz C, Sungur MA. Comparison of selective head cooling therapy and whole body cooling therapy in newborns with hypoxic ischemic encephalopathy: short term results. Turk Peadiatri Arsivi. 2015; 50(I): 27 - 36. https://doi.org//0.5152/tpa.2015.2167

18. Kali GT, Martinez-Biarge M, van Zyl J, Smith J, Rutherford M. Therapeutic hypothermia for neonatal hypoxic ischaemic encephalopathy had favourable outcomes at a referral hospital in the middle-income country. Acta Peadiatrica. 2016; 105(7): 806 - 15. https://doi.org/10.1 I I I/apa. 13392

19. Pin TW, Eldridge B, Galea MP. A review of developmental outcomes of term infants with post asphyxia neonatal encephalopathy. European Journal of Paediatric neurology. 2009; 13(3): 224 - 234. https://doi.org/I0.1016/j.ejpn.2008.05.00I

20. Shankaran S, Pappas A, McDonald SA, Vohr BR, Hintz SR, Yolton K, Gustafson KE, Leach TM, Green C, Bara R, Petrie Huitema CM, Ehrenkranz RA, Tyson JE, Das A, Hammond J, Peralta-Carcelen M, Evans PW, Heyne RJ, Wilson-Costello DE, Vaucher YE, Bauer CR, Dusick AM, Adams-Chapman I, Goldstein RF, Guillet R, Papile LA, Higgins RD, Eunice Kennedy Shriver NICHD Neonatal Research network. Childhood outcomes after hypothermia for neonatal encephalopathy. New England Journal of Medicine. 2012; 366: 2085 - 2092. https://doi.org/I0.1056/nejmoa I I I 2066

21. Shankaran S. Current status of hypothermia for hypoxic ischemia of the new born. Indian Journal Paediatrics. 20I4; 8I(16): 578 584. https://doi.org//0.1007/s | 2098-0|4-|468-6
22. Gardiner J, Wagh D, McMichael J, Hakeem M, Rao S. Outcomes of hypoxic ischaemic encephalopathy treated with therapeutic hypothermia using cool gel packs - experience from Western Australia. European Journal of Paediatric Neurology, 20 I4; 18(3): 39l - 8. https://doi.org//0.1016/j.ejpn.2014.02.003

23. Rosenbaum P, Paneth N, Leviton A, Goldstein M, Bax M. A report: The definition and classification of cerebral palsy. Developmental Medicine \& Child Neurology. 2007; 49: 8 - 14. https://doi.org/10.1 I I I/j. I469-8749.2007.tb I 2610.

24. Pallisano R, Rosenbaum P, Barlett D, Livingston M. GMFCS - E\&R, Gross Motor Functional Classification System Expanded and Revised. [cited November 20I I] Available from: http://www. canchild.com

25. Solarsh B, Katz B and Goodman M. START: Strive Towards Achieving Results Together. Johannesburg: Sunshine Centre Association; 1990.

26. Herbst I. Herbst Test Manual 2003.

27. Katzenellenbogen JM, Joubert G, Karim Abdool SS. Epidemiology. A manual for South Africa. Cape Town: Oxford University Press; 1997.

28. Horn AR, Swingler GH, Myer L, Harrison MC, Linley LL, Nelson C, Tooke L, Rhoda NR, Robertson NJ. Defining hypoxic ischemic encephalopathy in new born infants: benchmarking in a South African population. J Perinat. Med. 2013; 4I: 211 - 217. https://doi.org/10.1515/jpm-2012-0107

29. Le Doare K, Bland R, Newell M. Neurodevelopment in children born to HIV-infected mothers by infection and treatment status. Pediatrics. 20I2; 130(5): el326 - el346. https://doi.org// 0.1542/peds.20I2-0405

30. Munro SA, van Niekerk A, Seedat M. Childhood unintentional injuries: The perceived impact of the environment, lack of supervision and child characteristics. Child: Care, Health and Development. 2006; 32: 269 - 279. https://doi.org/I0.I I I I/j. I36522। 4.2006.00593.x

31. Mc Guiness C, Connolly P, Eakin A, Miller S. The Developmental Status of 2-3 year old Children entering Group-Based Settings in Northern Ireland. Belfast: Centre for Effective Education, Queen's University Belfast, 2012. [cited 2012]. Available from: http://www.early-years.org/news/news-info.php?newsid = 160

32. Sherry K, Draper CE. The relationship between gross motor skills and school readiness in early childhood: making the case in South Africa. Early Child Development and Care. 2012; 183(9): 1293 |3|0. https://doi.org//0.1080/03004430.2012.72/358

33. Barnhardt C, Borsting E, Deland P Pham N, Vu T. Relationship between visual-motor integration and spatial organization of written language and math. Optometry and Vision Science. 2005; 82(2): 138 - 143. https://doi.org//0.1097/01. opx.0000I53266.50875.53

34. Perez A, Ritter S, Brotschi B, Werner H, Caflisch J, Martin E, Latal B. Long-term neurodevelopmental outcome with HypoxicIschemic Encephalopathy. The Journal of Pediatrics. 2013; 163: 454 - 459.https://doi.org/10.1016/j.jpeds.2013.02.003

\section{AUTHOR CONTRIBUTIONS}

* Suzanne Stark worked at George Hospital when the study was initiated in 2010. Having completed her master's degree she took the lead in writing the protocol and first drafts of this article. She also contributed to doing assessments of children from 2010 until 2016 when she transferred to another place of work. She continued to provide input to the article up to the point where it was completed. 
Desiree Van Der Vyver worked at George Hospital as the Chief Occupational Therapist when the study was initiated. She was instrumental in capturing data and doing follow up assessments. She also provided crucial assistance with the writing of the article.

Pamela Gretschell is affiliated to the University of Cape Town. She provided unique guidance in the development of the protocol as well during the process of research. She also assisted with the authorship of the article.

Corresponding Author

Suzanne Stark

Email: sustark@gmail.com 